\title{
OPTIMAL LINEAR FILTERING OF GENERAL MULTIDIMENSIONAL GAUSSIAN PROCESSES AND ITS APPLICATION TO LAPLACE TRANSFORMS OF QUADRATIC FUNCTIONALS
}

\author{
M.L. KLEPTSYNA ${ }^{1}$ \\ Institute for Information Transmission Problems \\ Bolshoi Karetnii per. 19, Moscow 101447, Russia \\ E-mail: masha.kleptsyna@id.ru \\ A. LE BRETON \\ Université J. Fourier, Laboratoire de Modélisation et Calcul \\ BP 53, 38041 Grenoble Cedex 9, France \\ E-mail: alain.le-breton@imag.fr
}

(Received July, 2000; Revised March, 2001)

The optimal filtering problem for multidimensional continuous possibly non-Markovian, Gaussian processes, observed through a linear channel driven by a Brownian motion, is revisited. Explicit Volterra type filtering equations involving the covariance function of the filtered process are derived both for the conditional mean and for the covariance of the filtering error. The solution of the filtering problem is applied to obtain a Cameron-Martin type formula for Laplace transforms of a quadratic functional of the process. Particular cases for which the results can be further elaborated are investigated.

Key words: Gaussian Process, Martingale, Optimal Filtering, Filtering Error, Riccati-Volterra Equation, Cameron-Martin Formula.

AMS subject classifications: 60G15, 93E11, 60G44, 62M20.

\section{Introduction}

The Kalman-Bucy theory of optimal filtering is well-known for Gaussian linear systems driven by Brownian motions. Various extensions of this theory for possibly non-Gaussian Markov processes and semimartingales have been given a great deal of interest over the last decades. (see Davis [1], Liptser and Shiryaev [8, 9], Kallianpur [3], Elliot [3], and Pardoux [10]). As far as we know, there are few contributions for

\footnotetext{
${ }^{1}$ Research supported by RFBR Grants 00-01-00571 and 00-15-96116. 
systems generating non-Markovian processes or processes which are not semimartingales (e.g., [3]). Yet, for processes governed by for Itô-Volterra type equations, Kleptsyna and Veretennikov [7] provide a technique to overcome many of the difficulties of non-Markovian and non-semimartingale processes. Recently, a similar approach has been applied in several specific one-dimensional non-Markovian continuous Gaussian filtering problems (see Kleptsyna et al. [4-6], and references therein).

In this paper, we deal with a signal process $X=\left(X_{t}, t \geq 0\right)$ which is an arbitrary $p$-dimensional continuous Gaussian process and an observation process $Y=\left(Y_{t}, t \geq 0\right)$ in $\mathbb{R}^{q}$ governed by the linear equation

$$
Y_{t}=\int_{0}^{t} R(s) X_{s} d s+N_{t}, \quad t \geq 0
$$

(see [3, Chap. 10] for a similar setting). The function $R=(R(s), s \geq 0)$ is continuous with values in the set of $q \times p$ matrices, and $N=\left(N_{t}, t \geq 0\right)$ denotes a $q$-dimensional Brownian motion, independent of $X$, with covariance function $\langle N\rangle=\left(\langle N\rangle_{t}, t \geq 0\right)$. Clearly, the pair $(X, Y)$ is Gaussian but, in general, is neither Markovian nor a semimartingale. If only $Y$ is observed and one wishes to known $X$, the above reduces to the classical problem of filtering the signal $X$ at time $t$ from the observation of $Y$ up to time $t$. The solution to this problem is the conditional distribution of $X_{t}$ given the $\sigma$-field $\mathcal{Y}_{t}=\sigma\left(\left\{Y_{s}, 0 \leq s \leq t\right\}\right)$ which is called the optimal filter. Of course, here the optimal filter is a Gaussian distribution and it is completely determined by the conditional mean $\pi_{t}(X)$ of $X_{t}$ given $\mathcal{Y}_{t}$ and by the conditional covariances $\gamma_{X X}(t)$ of the filtering error, which is actually deterministic, i.e.,

$$
\pi_{t}(X)=\mathbb{E}\left(X_{t} / \mathcal{Y}_{t}\right) ; \gamma_{X X}(t)=\mathbb{E}\left(X_{t}-\pi_{t}(X)\right)\left(X_{t}-\pi_{t}(X)\right)^{\prime}
$$

Our first aim is to show that the solution can be completely described. That is, the characteristics of the optimal filter are obtained as the solution of a closed form system of Volterra-type equations which can be reduced to the Kalman-Busy equations when the signal process $X$ is a Gauss-Markov process. Our second aim is to extend the filtering approach for one-dimensional processes presented in [6], to obtain a Cameron-Martin type formula for the Laplace transform of a quadratic functional of the process. That is, for $q=p$,

$$
\mathcal{L}(t)=\mathbb{E} \exp \left\{-\frac{1}{2} \int_{0}^{t} X_{s}^{\prime} d\langle N\rangle_{s} X_{s}\right\} .
$$

This paper is organized as follows. In Section 2, we derive the solution of the filtering problems where explicit Volterra-type equations, involving the covariance function of the filtered process, are derived for the first and second-order moments of the optimal filter. The application to quadratic functionals of the process is reported in Section 3 where a filtering problem is given and the Laplace transform is computed. Finally, in Section 4, we investigate some specific cases where the results can be further elaborated. 


\section{Solution of the Filtering Problem}

In what follows, all random variables and processes are defined on the stochastic basis $\left(\Omega, \mathcal{F},\left(\mathcal{F}_{t}\right), \mathbb{P}\right)$ where the usual conditions are satisfied and where processes are $\left(\mathcal{F}_{t}\right)$ adapted. We consider a $\mathbb{R}^{p}$-valued continuous Gaussian process $X=\left(X_{t}, t \geq 0\right)$ with mean function $m=\left(m_{t}, t \geq 0\right)$ and covariance function $K=(K(t, s), t \geq 0, s \geq 0)$. That is,

$$
\mathbb{E} X_{t}=m_{t}, \quad \mathbb{E}\left(X_{t}-m_{t}\right)\left(X_{s}-m_{s}\right)^{\prime}=K(t, s), \quad t \geq 0, \quad s \geq 0
$$

For any process $Z=\left(Z_{t} ; t \in[0, T]\right)$ such that $\mathbb{E}\left|Z_{t}\right|<+\infty$, the notation $\pi_{t}(Z)$ is used for the conditional expectation of $Z_{t}$ given the $\sigma$-field $\mathcal{Y}_{t}$ :

$$
\pi_{t}(Z)=\mathbb{E}\left(Z_{t} / \mathcal{Y}_{t}\right)
$$

Here, we set $Q(s)=d\langle N\rangle_{s} / d s$ where the derivative is understood in the sense of absolute continuity. Thus $Q(s)$ is a non-negative symmetric $q \times q$ matrix assumed to be non-singular. Recall that the solution of the filtering problem of signal $X$ from observation $Y$ defined in (1) can be reduced to the equations for the conditional mean and covariance of the filtering error. The following theorem provides these equations.

Theorem 1: The conditional mean $\pi_{t}(X)$ and the covariance of the filtering error $\gamma_{X X}(t)$ defined by (2) are given by the equations

$$
\begin{gathered}
\pi_{t}(X)=m_{t}+\int_{0}^{t} \gamma(t, s) R^{\prime}(s) Q^{-1}(s)\left[d Y_{s}-R(s) \pi_{s}(X) d s\right], \quad t \geq 0, \\
\gamma_{X X}(t)=\gamma(t, t), \quad t \geq 0,
\end{gathered}
$$

where $\gamma$ is the solution of the Riccati-Volterra equation

$$
\gamma(t, s)=K(t, s)-\int_{0}^{s} \gamma(t, u) R^{\prime}(u) Q^{-1}(u) R(u) \gamma^{\prime}(s, u) d u, \quad 0 \leq s \leq t .
$$

Proof: The difficulty is that in general, $X$ is not a semimartingale. In order to apply the well-known filtering theory for semimartingales (see [2, 8, 9], for a fixed $t \geq 0$, we introduce the process $X^{t}=\left(X_{s}^{t}, 0 \leq s \leq t\right)$ as:

$$
X_{s}^{t}=\mathbb{E}\left[X_{t} / \sigma\left(\left\{X_{r}, 0 \leq r \leq s\right\}\right)\right], 0 \leq s \leq t .
$$

By definition, the process $X^{t}$ is a continuous martingale (with mean $m_{t}$ ) and $X_{t}^{t}=X_{t}$. Moreover, the pair $\left(X, X^{t}\right)$ is Gaussian and independent of $N$ so the distribution of $\left(X, X^{t}, Y\right)$ is still Gaussian. In particular, the conditional covariance $\gamma(t, s)=\mathbb{E}\left[\left(X_{s}^{t}-\pi_{s}\left(X^{t}\right)\right)\left(X_{s}-\pi_{s}(X)\right)^{\prime} / \mathcal{Y}_{s}\right]$ is deterministic. Hence, setting

$$
\delta_{X}(s)=X_{s}-\pi_{s}(X) \text { and } \delta_{X}^{t}(s)=X_{s}^{t}-\pi_{s}\left(X^{t}\right), \quad 0 \leq s \leq t
$$

we may write 


$$
\gamma(t, s)=\mathbb{E} \delta_{X}^{t}(s) \delta_{X}^{\prime}(s), \quad 0 \leq s \leq t .
$$

Since $X_{t}^{t}=X_{t}$ which implies that $\delta_{X}^{t}(t)=\delta_{X}(t)$, then for $s=t$, equality (7) reduces to equation (5).

We now introduce the innovation process $\nu=\left(\nu_{t}, t \geq 0\right)$ defined as

$$
\nu_{t}=Y_{t}-\int_{0}^{t} R(s) \pi_{s}(X) d s, \quad t \geq 0
$$

which plays a central role in general filtering theory (see [9]). Applying the fundamental filtering theorem to the pair of semimartingales $\left(X^{t}, Y\right)$, we immediately obtain

$$
\pi_{s}\left(X^{t}\right)=m_{t}+\int_{0}^{s} \gamma(t, r) R^{\prime}(r) Q^{-1}(r) d \nu_{r}, \quad 0 \leq s \leq t .
$$

Again, since $X_{t}^{t}=X_{t}$ and from definition (8), for $s=t$, equation (9) reduces to equation (4).

Therefore, to complete the proof of the first part of the theorem, we need only to show that function $\gamma$ defined by equation (7) is the solution of equation (6). From equation (9), and using equations (1) and (8), we can write

$$
\delta_{X}^{t}(s)=\left(X_{s}^{t}-m_{t}\right)-\int_{0}^{s} \gamma(t, r) R^{\prime}(r) Q^{-1}(r)\left[d N_{r}+R(r) \delta_{X}(r) d r\right], \quad 0 \leq s \leq t .
$$

Then, letting $0 \leq s \leq t$, we apply Itô formula to obtain the semimartingale decomposition of the process $\left(\delta_{X}^{t}(u)\left(\delta_{X}^{s}(u)\right)^{\prime}, 0 \leq u \leq s\right)$ :

$$
\begin{aligned}
& \delta_{X}^{t}(u)\left(\delta_{X}^{s}(u)\right)^{\prime}=\int_{0}^{u} \delta_{X}^{t}(r)\left\{d X_{r}^{s}-\gamma(s, r) R^{\prime}(r) Q^{-1}(r)\left[d N_{r}+R(r) \delta_{x}(r) d r\right]\right\}^{\prime} \\
& \quad+\int_{0}^{u} \delta_{X}^{s}(r)\left\{d X_{r}^{t}-\gamma(t, r) R^{\prime}(r) Q^{-1}(r)\left[d N_{r}+R(r) \delta_{X}(r) d r\right]\right\}^{\prime} \\
& +\left\langle X^{t}-m_{t}, X^{s}-m_{s}\right\rangle_{u}+\int_{0}^{u} \gamma(t, r) R^{\prime}(r) Q^{-1}(r) R(r) \gamma^{\prime}(s, r) d r
\end{aligned}
$$

Let us point out that due to the Gaussian property of the pair of martingales $\left(X^{t}, X^{s}\right)$, the bracket $\left\langle X^{t}-m_{t}, X^{s}-m_{s}\right\rangle_{u}$ is given by

$$
\left\langle X^{t}-m_{t}, X^{s}-m_{s}\right\rangle_{u}=\mathbb{E}\left(X_{u}^{t}-m_{t}\right)\left(X_{u}^{s}-m_{s}\right)^{\prime}
$$

and in particular, for $u=s$,

$$
\left\langle X^{t}-m_{t}, X^{s}-m_{s}\right\rangle_{s}=K(t, s) .
$$


Now let $u=s$ in equation (11) and compute the expectation of each side using the martingale property of $X^{t}, X^{s}$ and $N$ and definition (7). It is easy to check that $\gamma$ defined in (7) satisfies equation (6). This completes the proof of the theorem.

Remark 1: Theorem 1 provides further elaboration of the solution of the filtering problem given in [3, Chap. 10]. Theorem 1 can also be viewed as a partial extension to the non-Markovian setting of the filtering theorem for general linear systems driven by Gaussian martingales, as proved in Liptser and Shiryaev [9].

\section{The Cameron-Martin Type Formula}

Here, we start with a $p$-dimensional Gaussian process $X$, as before, and a given arbitrary increasing absolutely-continuous deterministic function $\langle N\rangle=\left(\langle N\rangle_{t}, t \geq 0\right)$ with values in the set of non-negative symmetric $p \times p$ matrices. We want to compute the Laplace transform $\mathcal{L}(t)$ defined by (3). Extending the filtering approach for one-dimensional processes given in [6], we can prove the following statement.

Theorem 2: For any $t \geq 0$, the following equality holds for the Laplace transform $\ell(t)$ defined in $(3)$ :

$$
\mathcal{L}(t)=\exp \left(-\frac{1}{2} \int_{0}^{t}\left[z^{\prime}(s) Q(s) z(s)+\operatorname{tr}(\gamma(s, s) Q(s))\right] d s\right),
$$

where $\gamma=(\gamma(t, s), 0 \leq s \leq t)$ is the unique solution of the Riccati-Volterra equation (6) with $Q(s)$ in place of $R^{\prime}(s) Q^{-1}(s) R(s)$, and $z=\left(z_{s}, s \geq 0\right)$ is the unique solution of the integral equation

$$
z_{s}=m_{s}-\int_{0}^{s} \gamma(s, u) Q(u) z_{u} d u, \quad s \geq 0
$$

The key point in the proof of this theorem is to describe an appropriate filtering problem of the type studied above and to extend the analysis beyond Theorem 1 . We take $q=p$ and we choose $N=\left(N_{t}, t \geq 0\right)$, with $N_{0}=0$, as a $\mathbb{R}^{p}$-valued Brownian motion with covariance function $\langle N\rangle$ that is independent of the given process $X$. We also choose $R(s)=Q(s)$, where, again, the notation $d\langle N\rangle_{s}=Q(s) d s$ is used, and we define the $\mathbb{R}^{p}$-valued observation process $Y+\left(Y_{t}, t \geq 0\right)$ by the corresponding equation (1), i.e.,

$$
Y_{t}=\int_{0}^{t} Q(s) X_{s} d s+N_{t}, \quad t \geq 0 \text {. }
$$

Finally, we define the auxiliary process $\xi=\left(\xi_{t}, t \geq 0\right)$ by

$$
\xi_{t}=\int_{0}^{t} X_{s}^{\prime} d Y_{s}, \quad t \geq 0
$$

and set 


$$
\gamma_{X \xi}(t)=\mathbb{E}\left[\left(X_{t}-\pi_{t}(X)\right)\left(\xi_{t}-\pi_{t}(\xi)\right) / \mathcal{Y}_{t}\right]
$$

We now state the following key result.

Lemma 1: For any $t \geq 0$, the following equality holds.

$$
\begin{aligned}
\mathcal{L}(t)=\exp \{- & \left.\frac{1}{2} \int_{0}^{t}\left(\pi_{s}(X)-\gamma_{X \xi}(s)\right)^{\prime} Q(s)\left(\pi_{s}(X)-\gamma_{X \xi}(s)\right) d s\right\} \\
& \times \exp \left\{-\frac{1}{2} \int_{0}^{t} \operatorname{tr}\left[Q(s) \gamma_{X X}(s)\right] d s\right\}
\end{aligned}
$$

Before presenting the proof of Lemma 1, it should be mentioned that equality (16) states that the difference $\pi_{s}(X)-\gamma_{X \xi}(s)$ is itself deterministic. Moreover, from a comparison of equations (12) and (16), it is clear from Lemma 1 that to prove Theorem 2, it is only necessary to show that the quantities $\gamma_{X X}(s)$ and $\pi_{s}(X)-\gamma_{X \xi}(s)$ are just $\gamma(s, s)$ and $z_{s}$, where $\gamma(s, s)$ and $z_{s}$ ar given by equations 96) with $R^{\prime}(s) Q^{-1}(s) R(s)$ replaced by $Q(s)$ and (13) respectively. These steps are now used to prove Lemma 1.

Proof of Lemma 1: It is easy to check that the function $\mathcal{L}$ is absolutely continuous and that the corresponding derivative is $-L / 2$, where

$$
L(t)=\mathbb{E} X_{t}^{\prime} Q(t) X_{t} e^{-I_{t}} ; \quad I_{t}=\frac{1}{2} \int_{0}^{t} X_{s}^{\prime} d\langle N\rangle_{s} X_{s} .
$$

Therefore, the following representation holds.

$$
\mathcal{L}(t)=\exp \left(-\frac{1}{2} \int_{0}^{t} \frac{L(s)}{\mathcal{L}(s)} d s\right)
$$

Now, for a fixed $t \geq 0$, define the random variable $\zeta_{t}$ by

$$
\zeta_{t}=\int_{0}^{t} X_{s}^{\prime} d N_{s}+\frac{1}{2} \int_{0}^{t} X_{s}^{\prime} d\langle N\rangle_{s} X_{s}
$$

Since $X$ and $V$ are independent, it is easy to check that $\mathbb{E} e^{-\zeta_{t}}=1$ thus we define the new probability $\widetilde{\mathbb{P}}_{t}=e^{-\zeta_{t}} \mathbb{P}$. The Girsanov Theorem states that $\left(\left(X_{s}, Y_{s}\right)\right.$, $0 \leq s \leq t)$ under $\widetilde{\mathbb{P}}_{t}$ (where $Y$ is given by $(10)$ ) and $\left.9\left(X_{s}, N_{s}\right), 0 \leq s \leq t\right)$ under $\mathbb{P}$ have the same distributions. Therefore, denoting the expectation computed with respect to $\widetilde{\mathbb{P}}_{t}$ by $\widetilde{\mathbb{E}}_{t}$, we obtain

$$
\mathcal{L}(t)=\widetilde{\mathbb{E}}_{t} e^{-I} ; \quad L(t)=\widetilde{\mathbb{E}}_{t} X_{t}^{\prime} Q(t) X_{t} e^{-I_{t}}
$$

In particular, since $X$ and $Y$ are independent under $\widetilde{\mathbb{P}}_{t}$, the above expectations can be replaced by the conditional expectations given $\mathcal{Y}_{t}$ under $\widetilde{\mathbb{P}}_{t}$, so that 


$$
\mathcal{L}(t)=\widetilde{\mathbb{E}}_{t}\left(e^{-I} t / \mathcal{Y}_{t}\right) ; \quad L(t)=\widetilde{\mathbb{E}}_{t}\left(X_{t}^{\prime} Q(t) X_{t} e^{-I} t / \mathcal{Y}_{t}\right)
$$

However, from Bayes formula, these equalities can be rewritten as

$$
\mathcal{L}(t)=\frac{\mathbb{E}\left(e^{-I_{t}} e^{-\zeta_{t}} / \mathcal{Y}_{t}\right)}{\mathbb{E}\left(e^{-\zeta_{t}} / \mathcal{Y}_{t}\right)} \text { and } L(t)=\frac{\mathbb{E}\left(X_{t}^{\prime} Q(t) X_{t} e^{-I_{t}} e^{-\zeta_{t}} / \mathcal{Y}_{t}\right)}{\mathbb{E}\left(e^{-\zeta_{t}} / \mathcal{Y}_{t}\right)}
$$

From definitions (1), (14) and (18), we have $\xi_{t}=I_{t}+\zeta_{t}$. Hence, it follows that

$$
\frac{L(t)}{\mathcal{L}(t)}=\frac{\mathbb{E}\left(X_{t}^{\prime} Q(t) X_{t} e^{-\xi_{t}} / \mathrm{Y}_{t}\right)}{\mathbb{E}\left(e^{-\xi_{t}} / \mathrm{Y}_{t}\right)}
$$

Now, observe that the joint distribution under $\mathbb{P}$ of $(X, Y)$ is Gaussian. Moreover, from equation (14), given $Y$ the variable $\xi_{t}$ for any $t \geq 0$ is a linear functional of $X$. Consequently, the conditional distribution of $\left(X_{t}, \xi_{t}\right)$ given the $\sigma$-field $\mathcal{Y}_{t}$ is also Gaussian. However, for a Gaussian pair $(U, V)$ in $\mathbb{R}^{p} \times \mathbb{R}$ and a non-negative $p \times p$ matrix $Q$, we have

$$
\frac{\mathbb{E} U^{\prime} Q U e^{-V}}{\mathbb{E} e^{-V}}=\operatorname{tr}\left[\gamma_{U U} Q\right]+\left[m_{U}-\gamma_{U V}\right]^{\prime} Q\left[m_{U}-\gamma_{U V}\right],
$$

where $m_{U}$ is the mean of $U$, and $\gamma_{U U}$ and $\gamma_{U V}$ are the covariances of $U$ and the cross covariance of $U$ and $V$ respectively. Therefore, from (19), we get

$$
\frac{L(t)}{\ell(t)}=\operatorname{tr}\left[\gamma_{X X}(t) Q(t)\right]+\left(\pi_{t}(X)-\gamma_{X \xi}(t)\right) Q(t)\left(\pi_{t}(X)-\gamma_{X \xi}(t)\right)^{\prime}
$$

Substituting this into (17) gives equation (16) and completes the proof of the lemma.

We now present the proof of Theorem 2.

Proof of Theorem 2: Note that since $R=R^{\prime}=Q$, in (6), the quantity $R^{\prime}(s) Q^{-1}(s) R(s)$ is just $Q(s)$. To complete the proof, we find $\pi_{s}(X)-\gamma_{X \xi}(s)$.

Using the complementary notation

$$
\delta_{\xi}(s)=\xi_{s}-\pi_{s}(\xi), \quad 0 \leq s \leq t
$$

we define

$$
\tilde{\gamma}(t, s)=\mathbb{E}\left(\delta_{X}^{t}(s) \delta_{\xi}(s) / \mathcal{Y}_{s}\right), \quad 0 \leq s \leq t
$$

Because $X_{t}^{t}=X_{t}$, we simply have $\gamma_{X \xi}(t)=\tilde{\gamma}(t, t)$. From (1) and (14) the process $\xi$ is a semimartingale with decomposition

$$
\xi_{t}=\int_{0}^{t} X_{s}^{\prime} Q(s) X_{s} d s+\int_{0}^{t} X_{s}^{\prime} d N_{s}
$$

Hence, the fundamental filtering theorem gives

$$
\pi_{t}(\xi)=\int_{0}^{t} \pi_{s}\left(X^{\prime} Q X\right) d s+\int_{0}^{t}\left[\pi_{s}(X)+\gamma_{X \xi}(s)\right]^{\prime} d \nu_{s}, \quad 0 \leq s \leq t .
$$


From the two previous equations and (8), it follows that for $0 \leq s \leq t$,

$$
\begin{gathered}
\delta_{\xi}(t)=\int_{0}^{t}\left(X_{s}^{\prime} Q(s) X_{s}-\pi_{s}\left(X^{\prime} Q X\right)\right) d s \\
-\int_{0}^{t} \delta_{X}^{\prime}(s) Q(s)\left(\pi_{s}(X)+\gamma_{X \xi}(s)\right) d s+\int_{0}^{t}\left(\delta_{X}(s)-\gamma_{X \xi}(s)\right)^{\prime} d N_{s}
\end{gathered}
$$

Using equations (10) and (20), applying the Itô formula to the process $\delta_{X}^{t} \delta_{\xi}$ and applying the fundamental filtering theorem, we obtain

$$
\begin{aligned}
\tilde{\gamma}(t, s) & =-\int_{0}^{s} \gamma(t, r) Q(r)\left[\tilde{\gamma}_{X \xi}(r)+\pi_{r}(X)\right] d r \\
& +\int_{0}^{s} \pi_{r}\left(\left(X^{\prime} Q X-\pi\left(X^{\prime} Q X\right) \delta_{X}\right) d r\right. \\
& +\int_{0}^{s}\left[\gamma(t, r)+\pi_{r}\left(\delta_{\xi} \delta_{X} \delta_{X}^{\prime}\right)\right] d \nu_{r} .
\end{aligned}
$$

Recall that the conditional distribution of $\left(X_{s}, \xi_{x}\right)$ given $\mathcal{Y}_{s}$ is Gaussian. But the third order centered moments of Gaussian distributions are equal to zero, and for the Gaussian pair $(U, V)$ in $\mathbb{R}^{p} \times \mathbb{R}$, and a non-negative $p \times p$ matrix $Q$, we have

$$
\mathbb{E}\left[U^{\prime} Q U-\mathbb{E} U^{\prime} Q U\right]\left[V-m_{V}\right]=2 \gamma_{U U} Q m_{V}
$$

Applying these properties and from (21), (4) and (8), we get

$$
\pi_{s}(X)-\tilde{\gamma}_{X \xi}(s)=m_{s}-\int_{0}^{s} \gamma(s, r) Q(r)\left[\pi_{r}(X)-\tilde{\gamma}_{X \xi}(r)\right] d r
$$

Thus, $\pi_{r}(X)-\tilde{\gamma}(r)=z$, where $z$ is the solution of equation (13) and so the proof of the proposition is complete.

\section{Particular Cases}

In the one-dimensional case, specific cases of Markovian and non-Markovian Gaussian processes for which the above results about filtering and Cameron-Martin type formulas can be applied, have been reported in [6] (see therein for further references of contributions around Laplace transforms of quadratic functionals). We now discuss some multidimensional examples where our results can be further elaborated. 


\subsection{Gauss-Markov Processes}

First we discuss the standard Gauss-Markov case where the $\mathbb{R}^{p}$-valued process $X$ is governed by the stochastic differential equation

$$
d X_{t}=A(t) X_{t} d t+d W_{t}, \quad t \geq 0 ; \quad X_{0}
$$

where $A=(A(t), t \geq 0)$ is a $p \times p$ matrix-valued continuous function, $W=\left(W_{t}, t \geq 0\right)$ is a Brownian motion in $\mathbb{R}^{p}$ such that $d\langle W\rangle_{t}=D(t) d t$, and $X_{0}$ is a Gaussian initial condition independent on $W$ such that $\mathbb{E} X_{0}=m$ and $\mathbb{E}\left(X_{0}-m\right)\left(X_{0}-m\right)^{\prime}=\Lambda$. Now, denote the solution of the differential equation $\dot{\Pi}_{s}=A(s) \prod_{s}, s \geq 0$, with the initial condition $\Pi_{0}=I_{p}(p \times p$ identity matrix $)$ by $\prod_{s}$. Then by $\prod_{s}$, we have

$$
m_{s}=\prod_{s} m, \quad K(t, s)=\prod_{t} \prod_{s}^{-1} K(s, s), \quad 0 \leq s \leq t,
$$

where $K(s, s)$ is a solution to the Lyapunov differential equation

$$
\frac{d}{d s} K(s, s)=A(s) K(s, s)+K(s, s) A^{\prime}(s)+D(s), s \geq 0, K(0,0)=\Lambda .
$$

In the filtering problem, it is well-known from the Kalman-Bucy theory that the covariance $\gamma_{X X}(s)$ of the filtering error is just the unique nonnegative solution of the Riccati differential equation

$$
\dot{\gamma}(s)=A(s) \gamma(s)+\gamma(s) A^{\prime}(s)-\gamma(s) R^{\prime}(s) Q^{-1}(s) R(s) \gamma(s)+D(s), 0 \leq s \leq t
$$

with initial condition $\gamma_{X X}(0)=\Lambda$. It then follows that the function $\gamma(t, s)$, where $\gamma(t, s)=\prod_{t} \prod_{s}^{-1} \gamma_{X X}(s)$ is the solution of equation (6) and that equation (4), for the conditional mean, can be reduced to the usual

$$
\begin{gathered}
d \pi_{s}(X)=A(s) \pi_{s}(X) d s+\gamma_{X X}(s) R^{\prime}(s) Q^{-1}(s)\left[d Y_{s}-R(s) \pi_{s}(X) d s\right] \\
s \geq 0, \quad \pi_{0}(X)=m .
\end{gathered}
$$

Now, concerning the Laplace transform $\mathcal{L}(t)$, we take $q=p$ and $R=Q$. Then the Riccati equation (23) for $\gamma_{X X}(s)$ reduces to

$$
\dot{\gamma}(s)=A(s) \gamma(s)+\gamma(s) A^{\prime}(s)-\gamma(s) Q(s) \gamma(s)+D(s), \quad 0 \leq s \leq t .
$$

Moreover, defining $Z=(Z(s), 0 \leq s \leq t)$ as the unique solution of the differential equation

$$
\dot{Z}(s)=\left[A(s)-\gamma_{X X}(s) Q(s)\right] Z(s), s \geq 0, \quad Z(0)=I_{p},
$$

it is readily seen that the function $z(s)$, where $z(s)=Z(s) m$, is the solution of the equation (13). Finally, inserting this into equation (12), we obtain

$$
\mathcal{L}(t)=\exp \left\{-\frac{1}{2} \int_{0}^{t}\left[m^{\prime} Z^{\prime}(s) Q(s) Z(s) m+\operatorname{tr}\left(\gamma_{X X}(s) Q(s)\right)\right] d s\right\} .
$$


Notice that in the present Gauss-Markov case, when $X_{0}=0$ (and hence $Z m \equiv 0$ ), Yashin [11] obtained an alternative expression of (25) using the backward Riccati equation instead of the forward equation (24). Actually, a direct link between these two representations can be shown without a probabilistic argument. This will be explained in a forthcoming paper where the link will be viewed within the scope of the usual mathematical duality between optimal control and optimal filtering.

\subsection{Iterated Integrals of a Brownian Motion}

Here we deal with the specific case of successive iterated integrals $J_{n}, n \geq 1$, of a onedimensional standard Brownian motion $B$, i.e., the processes $J_{n}, n \geq 1$ are defined for $n \geq 1$ and $t \geq 0$ by

$$
J_{0}(t)=B_{t} ; \quad J_{n}(t)=\int_{0}^{t} J_{n-1}(s) d s .
$$

Given a real number $\mu$, we want to compute the Laplace transform

$$
\ell_{n}(t ; \mu)=\mathbb{E} \exp \left\{-\frac{\mu^{2 n+2}}{2} \int_{0}^{t} J_{n}^{2}(s) d s\right\} .
$$

Of course, introducing the $(n+1)$-dimensional processes $W=(0, \ldots, 0, B)^{\prime}$ and $X=\left(J_{0}, \ldots, J_{n}\right)^{\prime}$, we can think of $J_{n}$ as the last component of the solution of the $(n+1)$-dimensional equation (22) with constant $(n+1) \times(n+1)$ matrices $A$ and $D$, where

$$
A=\left(\begin{array}{ccccc}
0 & 0 & \ldots & 0 & 0 \\
1 & 0 & \ldots & 0 & 0 \\
0 & 1 & \ldots & 0 & 0 \\
0 & 0 & \ldots & 1 & 0
\end{array}\right) ; \quad D=\left(\begin{array}{cccc}
1 & 0 & \ldots & 0 \\
. & . & \ldots & . \\
0 & 0 & \ldots & 0 \\
0 & 0 & \ldots & 0
\end{array}\right)
$$

and $X_{0}=0$ as the initial condition. Since $m=0$ (and hence $Z m=0$ ), $\Lambda=0$ and also

$\mu^{2 n+2} J_{n}^{2}=X^{\prime} Q_{\mu} X$, where $Q_{\mu}$ is the constant $(n+1) \times(n+1)$ matrix

$$
Q_{\mu}=\left(\begin{array}{cccc}
0 & 0 & \ldots & 0 \\
\cdot & . & \ldots & \cdot \\
0 & 0 & \ldots & 0 \\
0 & 0 & \ldots & \mu^{2 n+2}
\end{array}\right)
$$

Then from (25), we get

$$
\ell_{n}(t ; \mu)=\exp \left\{-\frac{1}{2} \int_{0}^{t} \operatorname{tr}\left(\gamma_{\mu}(s) Q_{\mu}\right) d s\right\}
$$


where, because of (24), $\gamma_{\mu}$ is the solution of the Riccati equation

$$
\dot{\gamma}_{\mu}(s)=A \gamma_{\mu}(s)+\gamma_{\mu}(s) A^{\prime}-\gamma_{\mu}(s) Q_{\mu} \gamma_{\mu}(s)+D, \quad 0 \leq s \leq t ; \quad \gamma_{\mu}(0)=0 .
$$

We apply the linearization method to this equation and define the pair $\left(\Delta_{\mu}(s), \nabla_{\mu}(s)\right)$ of $(n+1) \times(n+1)$ matrices as the solution of the differential system

$$
\left(\dot{\Delta}_{\mu}(s), \dot{\nabla}_{\mu}(s)\right)=\left(\Delta_{\mu}(s), \nabla_{\mu}(s)\right) \Gamma_{\mu} ; \quad\left(\Delta_{\mu}(0), \nabla_{\mu}(0)\right)=(I, 0),
$$

where

$$
\Gamma_{\mu}=\left(\begin{array}{cc}
-A & D \\
Q_{\mu} & A^{\prime}
\end{array}\right)
$$

Then $\gamma_{\mu}=\Delta_{\mu}^{-1} \nabla_{\mu}$ and, since $\operatorname{tr}(A)=0$,

$$
\begin{gathered}
\int_{0}^{t} \operatorname{tr}\left(\gamma_{\mu}(s) Q_{\mu}\right) d w=\int_{0}^{t} \operatorname{tr}\left(\Delta_{\mu}^{-1}(s) \dot{\Delta}_{\mu}(s)\right) d s \\
=\log \operatorname{det}\left(\Delta_{\mu}(t)\right) .
\end{gathered}
$$

Observing that $\Gamma_{\mu}^{2 n+2}=(-1)^{n} \mu^{2 n+2} I_{2 n+2}$, it is easily checked that $\Delta_{\mu}(t)=$ $\Delta_{1}(\mu t)$ where $\delta$ is the solution of the $(2 n+2)$-th order differential equation

$$
\delta^{(2 n+2)}(s)=(-1)^{n} \delta(s) ; \quad \delta(0)=1, \quad \delta^{(k)}(0)=0, \quad k=1, \ldots, 2 n+1,
$$

and the $(i, j)$-entry of $\Delta_{1}$ is given by

$$
\delta_{i j}=\left\{\begin{array}{cc}
(-1)^{j-i} \delta^{(j-i)}, & j \geq i \\
(-1)^{n+i-j} \delta^{(2 n+2-i+j)}, & i>j
\end{array}\right.
$$

Finally, the function $\delta$, which is just

$$
\delta(t)=\frac{1}{2 n+2} \sum_{i=1}^{2 n+2} e^{z 2 n+2, i^{t}}
$$

where the $z_{2 n+2, i}$ 's are the $2 n+2$ roots of the equation $z^{2 n+2}=(-1)^{n}$, allows the representation

$$
\ell_{n}(t ; \mu)=\mathscr{L}_{n}(\mu t ; 1), \quad \mathcal{L}_{n}(t ; 1)=\left[\operatorname{det}\left(\Delta_{1}(t)\right)\right]^{-1 / 2}
$$

For example, taking, $n=1$, for the integral $J_{1}(t)=\int{ }_{0}^{t} B_{s} d s$, it can be seen that

$$
\mathbb{E} \exp \left\{-\frac{\mu^{4}}{2} \int_{0}^{t} J_{1}^{2}(s) d s\right\}=\sqrt{2}\left\{\cosh ^{2} \frac{\mu}{\sqrt{2}} t+\cos ^{2} \frac{\mu}{\sqrt{2}} t\right\}^{-1 / 2} .
$$




\section{Acknowledgements}

We are very grateful to Michel Voit for valuable discussions and for bringing book [3] to our attention.

\section{References}

[1] Davis, M.H.A., Linear Estimation and Stochastic Control, Chapman and Hall, New York 1977.

[2] Elliott, R.J., Stochastic Calculus and Applications, Springer-Verlag, New York 1982.

[3] Kallianpur, G., Stochastic Filtering Theory, Springer-Verlag, New York 1980.

[4] Kleptsyna, M.L., Kloeden, P.E. and Ahn, V.V., Linear filtering with fractional Brownian motion, Stoch. Anal. and its Appl. 16:5 (1998), 907-914.

[5] Kleptsyna, M.L., Le Breton, A. and Roubaud, M.-C., An elementary approach to filtering in systems with fractional Brownian observation noise, In: Prob. Theory and Math. Stat. Proc. of the 7th Vilnius Conf. (ed. by B. Grigelionis et al.), VSP/TEV (1999), 373-392.

[6] Kleptsyna, M.L. and Le Breton, A., A Cameron-Martin type formula for general Gaussian processes - A filtering approach, Stoch. and Stoch. Reports (2001), to appear.

[7] Kleptsyna, M.L. and Veretennikov, A. Yu., Linear filtering and properties of conditional laws of Itô-Volterra equations, Stats. and Control of Stoch. Proc. (1985), 179-196.

[8] Liptser, R.S. and Shiryaev, A.N., Statistics of Random Processes, SpringerVerlag, New York 1978.

[9] Liptser, R.S. and Shiryaev, A.N., Theory of Martingales, Kluwer Academic Pub., Dordrecht 1989.

[10] Pardoux, E., Filtrage non linéaire et équations aux dérivées partielles stochastiques associées, In: Ecole d'été de Probabilités de Saint-Flour XIX - 1989 (ed. by P.L. Hennequin), Lecture Notes in Math, Springer Verlag 1464 (1991), 67163.

[11] Yashin, A.I., An extension of the Cameron-Martin result, J. Appl. Probab. 30:1 (1993), 247-251. 


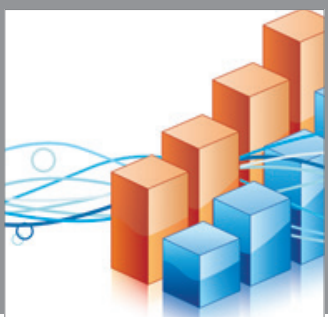

Advances in

Operations Research

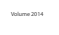

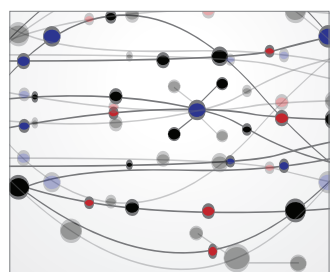

\section{The Scientific} World Journal
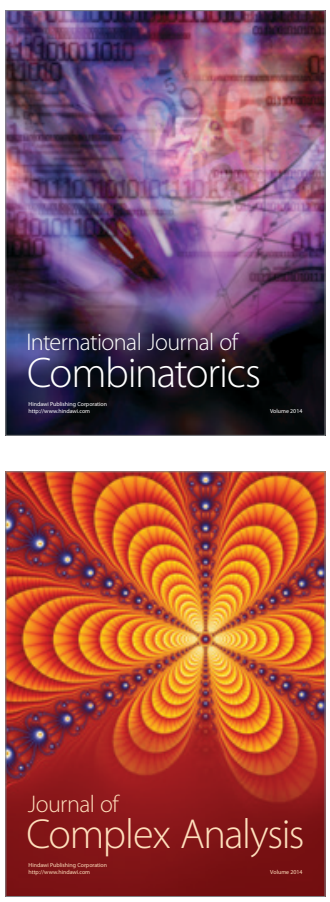

International Journal of

Mathematics and

Mathematical

Sciences
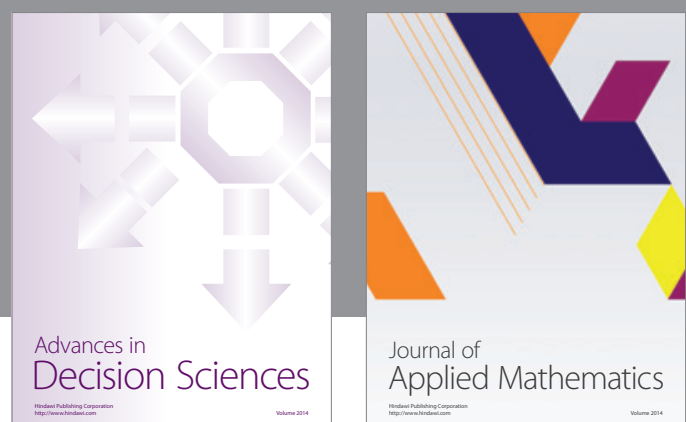

Journal of

Applied Mathematics
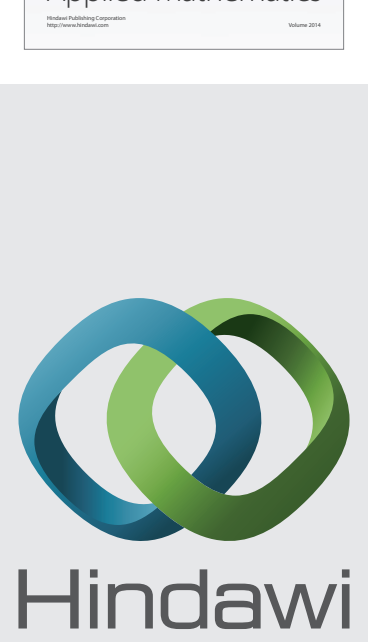

Submit your manuscripts at http://www.hindawi.com
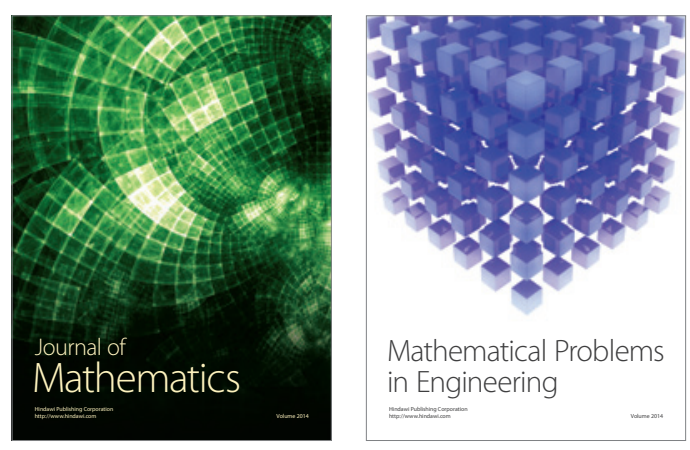

Mathematical Problems in Engineering
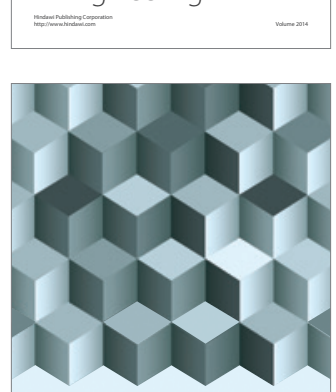

Journal of

Function Spaces
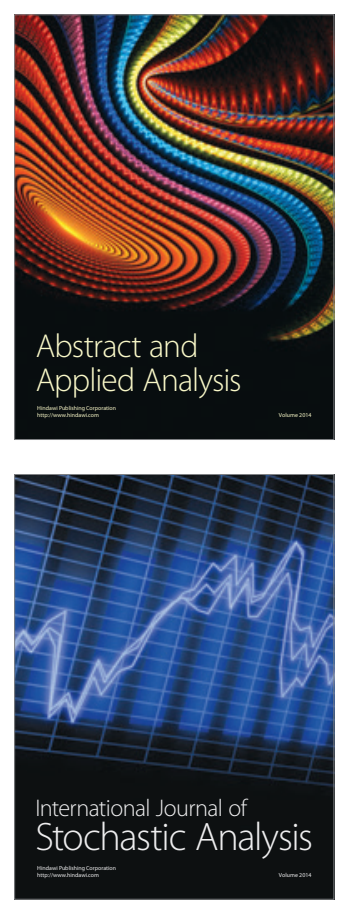

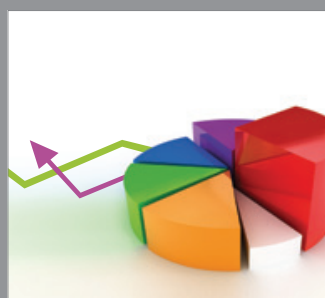

ournal of

Probability and Statistics

Promensencen
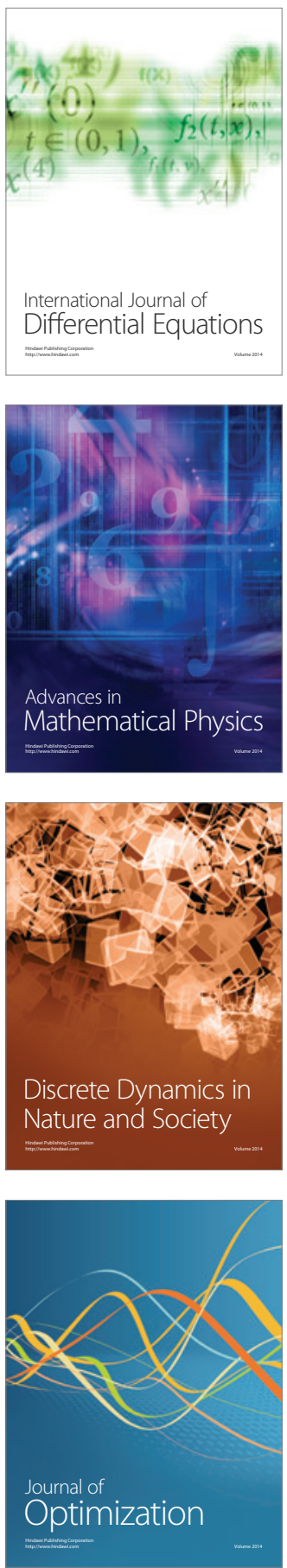